\title{
The effects of probiotics and oxytocin nasal spray on neuro-social behaviors of children with Autism Spectrum Disorders (ASD): study protocol for a randomized, double-blinded, placebo-controlled, parallel-group clinical trial
}

Xue-Jun Kong ( $\nabla$ xkong1@mgh.harvard.edu )

Massachusetts General Hospital

Jun Liu

Massachusetts General Hospital

Jing Li

Indiana University

Kenneth Kwong

Massachusetts General Hospital

Madelyn Koh

Massachusetts General Hospital

Piyawat Sukijthamapan

Harvard Medical School

Jason J. Guo

Northeastern University

Zhenyu Jim Sun

Harvard Medical School

Yiqing Song

Indiana University

Method Article

Keywords: Autism spectrum disorders; Intranasal; Oxytocin; Probiotics

Posted Date: January 31st, 2019

DOI: https://doi.org/10.21203/rs.2.260/v1

License: (c) (i) This work is licensed under a Creative Commons Attribution 4.0 International License.

Read Full License 


\section{Abstract}

Background: Autism spectrum disorder (ASD) is a complex neurodevelopmental disorder characterized by impairments in social interaction and communication. Oxytocin (OXT), as a neuropeptide, plays a role in emotional and social behaviors. Animal studies showed that Lactobacillus reuteri (L. reuteri) supplementation led to an OXT-dependent behavioral improvement in ASD mouse models. We thus designed the first clinical trial to test whether supplementation with L. reuteri would improve social behavioral functions in ASD patients; whether there is a synergy between probiotics treatment and exogenous OXT therapy; and whether such OXT pathway-targeted therapies alter the gut-brain axis, including autonomic function, gastrointestinal (GI) health, gut microbial profiles, gut/brain inflammation, and brain activity.

Methods/design: This clinical trial is a two-staged, randomized, double-blind, placebo-controlled, parallelgroup study. Throughout the study (0-24 weeks), 60 ASD patients will be randomly assigned to receive either oral L. reuteri probiotics or placebo. In the 2nd stage (13-24 weeks) of the study, all participants will also receive intranasal OXT spray. As primary outcomes, serum OXT levels will be assayed and social behaviors will be measured via two validated questionnaires, an objective emotional facial matching test, and a new video-based eye tracking test. Secondary outcomes include autonomic function indices, GI function, gut microbiome/short chain fatty acids, brain activity in social response regions via functional magnetic resonance imaging (fMRI), and serum neuroinflammatory/neurotransmitter/neuro-injury biomarkers. All the outcomes will be assessed at baseline, week 12, and week 24.

Discussion: This pilot study will provide important empirical data to evaluate potential effects of oral probiotics in improving ASD core symptoms through modulating endogenous OXT, examine the synergistic effects between probiotics and exogenous OXT treatment, and further explore the relationship of OXT with the gut-brain axis in ASD patients.

Trial registration: ClinicalTrial.gov, NCT03337035. Registered 8 November 2017, https://clinicaltrials.gov/ct2/show/NCT03337035

Keywords: Autism spectrum disorders; Intranasal; Oxytocin; Probiotics

\section{Background}

Autism spectrum disorder (ASD) is a complex neurological and developmental disorder characterized by impaired communication and social interaction skills, as well as stereotypical repetitive behavioral patterns ${ }^{1}$. In the United States of America (USA), the prevalence of ASD has nearly tripled from 1 in 150 as of 2000 to 1 in 59 as of $2018^{1}$. Its etiology remains elusive, but some pathophysiological findings may shed light on its effective treatment ${ }^{2-4}$. 
Oxytocin (OXT) is a hypothalamic neuropeptide of multiple functions, including modulation of emotional and social communication, bonding, and reward-related behaviors ${ }^{5}$. In murine studies, mice with a deficiency of OXT signaling (OXT ligand or receptor knockout and genetically deficient oxytocin exocytosis models) all displayed autistic like behavior ${ }^{6,7}$. There is also some evidence from humans showing OXT deficiency in ASD subjects ${ }^{8}$. Thus, dysfunction of OXT signaling has been implicated to play an important role in the etiology of $A S D^{9}, 10$. As an easily administered and cost-effective treatment with possibly minimal adverse effects, OXT holds promising therapeutic potential in treating ASD core symptoms ${ }^{11}$. Andari et al. reported that in a small randomized placebo-controlled trial, 13 patients with high functioning autism had improved social recognition and attention to social cues after OXT administration $^{12}$. A meta-analysis of 12 randomized controlled trials (RCT) of OXT on social recognition of ASD patients showed no evidence of significant efficacy of OXT compared to placebo ${ }^{11}$; however, the level of such overall evidence was low due to substantial between-trial heterogeneity, including variations in study design, patient characteristics, primary outcome assessments, and OXT dosage and duration. In addition, ASD patients may vary in the functionality of their endogenous OXT system, and it is possible that only the subgroups that are more genetically prone to OXT signaling dysfunction may respond well to OXT treatments.

Of note, OXT signaling may be a key link in the gut-brain-axis and seems inducible by certain probiotics. Poutahidis et al. ${ }^{13}$ first described the induction of endogenous OXT signaling by probiotics L. reuteri in animal studies; subsequent animal studies provided consistent evidence of OXT-dependent behavioral improvement in multiple ASD mouse models after supplementation with the L. reuteri ${ }^{14-16}$. Previous studies showed that probiotics reduced responses to negative emotional stimuli in amygdala, insula and fronto-limbic regions, compared with placebo ${ }^{17,} 18$. However, none of the published studies has reported the efficacy of L. reuteri in ASD patients. Further, there is no studies that specifically examine the synergistic effects between L. reuteri and OXT therapy.

To address these questions, we developed our central hypothesis that supplementation with probiotics ( $\mathrm{L}$. reuteri), independently or in combination with intranasal OXT treatment, would lead to improvement of social and behavioral functions in ASD patients via induction of OXT signaling. We thus designed a twostaged randomized clinical trial to test the hypothesis. Specifically, we proposed the following two primary aims and a secondary aim.

Primary aim 1: To investigate the efficacy of L. reuteri alone vs. placebo in treating ASD core symptoms. Primary outcomes include changes of socio-behavioral scores and serum OXT levels between baseline and 12 weeks.

Primary aim 2: To examine the efficacy of L. reuteri plus OXT therapy in treating ASD core symptoms, and explore whether the efficacy of dual therapy depends on OXT signaling. Primary outcomes include changes in socio-behavioral scores and serum OXT levels between 12 and 24 weeks. 
Secondary aim: To examine whether L. reuteri therapy alone or L. reuteri plus OXT therapy modulate the OXT signaling/gut-brain-axis. Our secondary outcomes include changes in the following variables between baseline and 12 week and between 12 and 24 week: brain activity in social response region measured by $\mathrm{fMRI}$, autonomic function indices, the gut dysbiosis/GI dysfunction using questionnaires and microbiome profiling, and serum neuroinflammatory/neurotransmitter/neuroinjury biomarkers as described below.

We believe that this study will provide useful information on the efficacy of $\mathrm{L}$. reuteri single therapy and $\mathrm{L}$. reuteri plus OXT dual therapy for targeting the ASD core symptoms. Furthermore, it will provide important preliminary data to evaluate the mechanisms of how L. reuteri and OXT affect the gut-brain-axis in human subjects, and the utility of biomarkers to predict treatment responses to L. reuteri and L. reuteri and OXT dual therapy.

\section{Methods}

To address our primary and secondary aims, we design a two-staged, randomized, double-blinded, placebo-controlled, parallel-group clinical trial. In the first stage of the trial, we will randomly assign 60 patients, in a 1:1 ratio, to either $L$. reuteri treatment or placebo in order to assesses the effects of 12-week administration of L. reuteri alone on the behavioral measurements of ASD, and whether treatment response depends on the degree of baseline OXT level and/or endogenous OXT signaling induction by probiotics. In the second stage of the trial, we will add intranasal OXT to both groups while their L. reuteri or placebo treatment continue for an additional 12 weeks to explore the possibility of synergy between exogenous intranasal OXT and endogenous OXT induction by L. reuteri. We anticipate that a 12-week treatment duration will be sufficient for $L$. reuteri supplementation to induce detectable changes in OXT, ut microbiome, and social awareness/behavioral scores.

This study description is in accordance with the Consolidated Standards of Reporting Trials (CONSORT) guidelines that are published for the evaluation of randomized controlled trials. The trial will be overseen by an independent trial steering committee and a separate data and safety monitoring committee. All the participants will provide written informed consent. The study design, including the screening, randomization, and follow-up of ASD patients, was approved by Massachusetts General Hospital (IRB protocol number: 2017P001667).

\section{Participants}

All the participants should meet the inclusion criteria as follows:

1) Age between 3-25 years old;

2) Pre-existing diagnosis of autism and confirmation of diagnosis by Diagnostic and Statistical Manual of Mental Disorders (DSM)-V-TR criteria, Autism Diagnostic Observation Schedule (ADOS) and/or Autism Diagnostic Interview-Revised (ADI-R); 
3) Being able to attend clinic visits;

4) Stable medications for at least 4 weeks;

5) No planned changes in medications or psychosocial interventions during the trial;

6) Willingness to provide blood samples.

Participants with any of the following conditions will be excluded:

1) Pregnant or breast-feeding woman (before or during the study);

2) Comorbidity of other neurological and/or psychiatric disorders, including unstable seizure, schizophrenia, schizoaffective disorder, bipolar disorders, or history of substance abuse;

3) History of oxytocin, antibiotics or probiotic use in the month prior to enrollment;

4) Subjects with active cardiovascular disease that is not controlled by medication;

5) Regular nasal obstruction or nosebleeds;

6) Significant hearing or vision impairments;

7) Habitual consumption of large volumes of water.

Participants who are selected to undergo fMRI examinations must have the ability to lay flat and motionless for up to 1 hour. Participants are excluded from the fMRI study if any of the following are not met:

1) Any contraindications to fMRI scanning, such as electrically activated implanted devices, other ferromagnetic implants, and certain tattoos;

and 2) Claustrophobia.

Patients taking psychotropic medications will be asked to keep their medication doses constant throughout the trial.

ASD diagnostic criteria: Diagnostic and Statistical Manual of Mental Disorders (DSM)-V-TR is published by the American Psychiatric Association, and offers a common language and standard criteria for the classification of mental disorders. It is used, or relied upon, by clinicians, researchers, psychiatric drug regulation agencies, health insurance companies, pharmaceutical companies, the legal system, and policy makers together with alternatives such as the ICD-10 Classification of Mental and Behavioral Disorders, produced by the WHO. The Autism Diagnostic Observation Schedule (ADOS) is a semistructured assessment of communication, social interaction, and play (or imaginative use of materials) for individuals suspected of having autism or other pervasive developmental disorders. The Autism 
Diagnostic Interview-Revised (ADI-R) is a structured interview conducted with the parents of individuals who have been referred for the evaluation of possible autism or autism spectrum disorders.

\section{Randomization and Blinding}

Randomization and allocation concealment will be performed in collaboration with Massachusetts General Hospital (MGH) research pharmacy. Randomization sampling numbers will be electronically generated, and central randomization at the research pharmacy using coded drug containers of identical appearance prepared by the research pharmacy will ensure allocation concealment. Blinding will be maintained by making the capsules look identical. Both participants and the research staff who collect the outcome data will be blinded to treatment status.

\section{Recruitment and Consent}

ASD patients will be recruited from primary care, pediatric, and ASD clinics at MGH, Beth Israel Deaconess Medical Center, community ASD education events, and charity ASD programs in Boston as well as international participants. Screening will be performed by trained staff members via phone calls at A. Martinos Center for Biomedical Imaging, MGH. Informed consent will be obtained in-person at the initial study visit, and minors will require assent and consent of at least one parent or legal guardian.

\section{Study procedure}

The treatment will proceed for a total of 24 weeks (Figure 1 and 2). In the first stage (12 weeks), all the patients will be randomly assigned to two groups: Group A (30 subjects) receives oral probiotics while Group B (30 subjects) receives oral as a placebo. In the second stage, subjects in Group A and Group B will continue their respective oral probiotics or placebo administration as in stage 1 . In addition, both groups will be simultaneously administered with intranasal OXT spray for an additional 12 weeks.

\section{Intervention}

First stage: probiotic bacteria, L. reuteri (BioGaia), will be used in the study. It has been used in previous clinical trials, including those targeting pediatric populations ${ }^{19-21}$. Subjects will receive $10^{10}$ colonyforming units of L. reuteri daily or placebo for a duration of 3 months. L. reuteri has demonstrated consistently favorable safety profiles across multiple clinical trials ${ }^{19-21}$.

Second stage: We choose Syntocinon intranasal oxytocin spray (Novartis pharmaceuticals) as it is currently the most commonly used standardized oxytocin nasal spray for clinical trials worldwide. We will instruct the patient and family members to the use of spray, which begins with 1 puff of $4 \mathrm{IU}$ daily. After 1 week, the dose will increase to 1 puff per nostril for both nostrils daily $(8 \mathrm{IU} / \mathrm{d})$. After the second week, the dose will increase to 1 puff per nostril for both nostrils twice a day $(16 \mathrm{IU} / \mathrm{d})$. After the third week, the dose will titrate up to the maximum dose $24 \mathrm{IU}$ daily, which is 2 puffs per nostril for both nostrils in the morning, and 1 puff per nostril for both nostrils in the afternoon. The dosage of $24 \mathrm{IU}$ per day has been approved safe and adequate in even younger patients (age 3-8 years old) by previous publications 22,23 . 


\section{Outcomes}

Primary outcomes:

1. Social and behavioral scores assessed using behavioral assessment questionnaires, including the Aberrant Behavior Checklist (ABC) and Social Responsiveness Scale-2 (SRS-2). ABC is a 58-item behavior rating scale used to measure behavior problems across five subscales, which include irritability, lethargy/social withdrawal, stereotypic behavior, hyperactivity/noncompliance, and inappropriate speech. SRS-2 consists of 65 items used for quantitative assessment of the severity of ASD symptoms. The SRS-2 (Constantino and Gruber 2005) is a caregiver completed rating scale assessing social interest and interaction. Scores from SRS-2 and ABC are both continuous, including composite summary scores and subscale scores (ABC: composite score scale $=0-174$, social withdraw subscale $=0-48$, stereotypical behavior subscale $=0-21$, inappropriate speech subscale $=0-12$; SRS-2: composite score scale $=0-195$, social awareness subscale $=0-24$, social cognition subscale $=0$ 36 , social communication subscale $=0-66$, social motivation subscale $=0-33$, autistic mannerism subscale=0-36).

2. Objective ASD behavioral scores assessed by using eye tracking test and emotional face matching tests. For eye tracking test, fixation time for the eye and body area (continuous variable) will be calculated for data analysis (ASD reference range 0-2000 ms) ${ }^{24}$. For emotional face matching test, performance measures consist of reaction time (RT) (ASD reference range: $8.18 \pm 3.42 \mathrm{~s}$ ) and accuracy (ASD reference range: $62.5 \pm 21.0 \%)^{25}$.

3. Baseline levels and increment of serum OXT levels by probiotics (ASD reference range: 31.77-314.35 $\mathrm{pg} / \mathrm{ml})^{8}$.

Secondary outcomes:

1. Gastrointestinal $(\mathrm{GI})$ health indices measured by validated $\mathrm{GI}$ symptoms assessment questionnaires (GSI scores and Bristol Stool Chart). The GI function scores are ordinal variables (scale: 0-17).

2. Gut microbiome/short chain fatty acids profiles; gut microbiome composition is presented as relatively abundance of different bacterial taxa (scale: 0-100\%).

3. Oxytocin-related neurotransmitter levels (GABA, dopamine, and serotonin), inflammatory cytokine levels (IL-1 beta, IL-6, TGF-beta, and interferon-gamma), neuroinjury serum markers (S100, GFAP, and $\mathrm{MBP}$ ), autonomic neurotransmitter levels (epinephrine and norepinephrine).

4. Autonomic indices including blood volume pulse, heart rate variation, peripheral skin temperature, skin electrodermal activity, and 3-axis motion accelerometer.

5. Brain activity in social response regions measured by $\mathrm{fMRI}$. $\mathrm{fMRI}$ indices include beta-values of Blood Oxygen Level Dependent (BOLD) activities, functional connectivity and figure plethysmography during emotional task, resting-state and breath-hold. 
The primary and secondary outcomes are the same for the first stage of the trial (probiotics alone, 0-12 weeks), and the second stage of the trial (probiotics plus OXT dual therapy, 0-24 weeks). All questionnaires and tests will be conducted 3 times total at week 0, week 12, and week 24 (prior to treatment and upon completion of the first and second stage of therapy).

\section{Data Collection}

All data collection will be performed at 0 week (baseline), 12 weeks (end of probiotics-only stage), and 24 weeks (end of probiotics plus OXT dual therapy stage). Study data will be collected and managed using REDCap electronic data capture tools hosted at Massachusetts General Hospital. REDCap (Research Electronic Data Capture) is a secure, web-based application designed to support data capture for research studies, providing 1) an intuitive interface for validated data entry; 2) audit trails for tracking data manipulation and export procedures; 3 ) automated export procedures for seamless data downloads to common statistical packages; and 4) procedures for importing data from external sources.

Baseline information: we will collect information regarding patients' past medication history, immune functions, and lifestyle factors affecting the gut microbiome using questionnaires.

ASD behavior assessment: ASD-related behavioral symptoms will be assessed by SRS-2 and ABC, which are two validated behavioral symptoms questionnaires widely used in ASD-related clinical trials. The SRS-2 is a caregiver completed rating scale assessing social interest and interaction. The scale provides a dimensional measure of social interaction allowing the rating of social skills in autism as well as nonautistic individuals. $A B C$ is an informant rating instrument which resolves into five subscales, including irritability, lethargy/social withdrawal, stereotypic behavior, hyperactivity/noncompliance, and inappropriate speech.

Eye tracking test: We will use a new video-based eye tracking device developed by Kong et al. ${ }^{26}$ to objectively assess participants' attention to human body parts. The paradigm consists of 10 video scenarios. Study subjects will be seated and allowed to watch a video containing moving geometric patterns on one side of an eye tracking monitor and activities of human subjects on the other. The eye tracking device records subjects' eye gaze and characterize the probability of the subject concentrating on geometric shapes or human subjects.

Emotional facial matching test: We will use a previously validated emotional facial matching paradigm to objectively assess ASD patients' ability to detect and characterize human emotions ${ }^{25}$. In this task, participants have to recognize a low-intensity and therefore ambiguous facial expression. Four expressions were used: happy, fearful, angry and neutral. Dynamic morphs were created from the NimStim Emotional Face Stimuli database (http://www.macbrain.org/faces/index.htm\#faces) between NEUTRAL and each EMOTIONAL expression using Morph Age Pro (http://www.creaceed.com/morphage/), and still images were created at $40 \%$ intensity level between neutral and the full emotional expressions. Participants will be then presented with one still image depicting happy, fearful or angry at $40 \%$ intensity or NEUTRAL on the left side of the computer screen, 
while on the right still Ekman stimuli representing each of the four full emotional expressions were shown.

Functional Magnetic Resonance Imaging (fMRI): A subsample from the trial participants, 10 ASD patients in each arm, will be randomly chosen for fMRI examination. We will measure brain activity in areas related to emotional processing at baseline and upon specific tasks involving facial emotion recognition following the protocol of Hadjikhani et al. $2015^{25}$. This protocol was developed for an ASD drug trial and it measures brain activity for neutral and emotional faces at baseline and after treatment and compares the difference of activity for [emotion vs. neutral - after] minus [emotion vs neutral - before]. Reference range is not available because we will be using a protocol designed de novo for this study. The fMRI will be done in-house at the A Martinos Center for Biomedical imaging at MGH. Several procedures will be in place to protect the patient from any risk associated with the MRI scanning environment.

GI health assessment: GI symptoms will be assessed by validated GI severity index (GSI) and Bristol Stool Chart; GI microbiome and metabolites and Short Chain Fatty Acid profiling will be performed at Microbiome core facility at Brigham and Women's Hospital (Boston, MA). To quantify and analyze gut microbiome, $16 \mathrm{~S}$ sequencing data will be processed and analyzed with QIIME software package v. 2018.2.0. Alpha diversity will be calculated on the basis of the gene profile for each sample based on the Shannon index, Faith's index, and Simpson's evenness index. Beta diversity will be calculated on the unweighted and weighted UniFrac distances, Jaccard and the Bray-Curtis dissimilarity.

Autonomic function assessment: Autonomic indices will be performed by E4 Empatica wristband (https://www.empatica.com/science). This is a commercially available research-grade wearable device that measure autonomic indices include blood volume pulse (BVP), heart rate variability (HRV), peripheral skin temperature, skin electrodermal activity (EDA), and 3-axis motion accelerometer at resting state and upon application of external anxiety-provoking stimuli. From the raw autonomic indices data, we will create a stress test algorithm for all autonomic indices gathered from the wristband which will compare the subject's physiological stress before, leading up to, during, and after a stress stimulus.

\section{Measurements of serum levels of inflammatory/neurotransmitter/neuroinjury biomarkers: serum} samples will be collected through phlebotomy at 0 week (baseline), 12 weeks (end of probiotics-only stage), and 24 weeks (end of probiotics and OXT dual therapy stage). Serum levels of OXT, GABA, Dopamine, Serotonin, IL-1beta, IL-6, TGF-beta, interferon-gamma, MBP, S100, GFAP, epinephrine and norepinephrine will be measured by Partners Healthcare clinical research laboratory core facility, which has performed rigorous internal quality control and validation of the assays with acceptable inter and intra-individual coefficient of variation. All potential biomarkers testing will be done in labs within the Partners System labeled as research subjects without full identification of the subjects.

\section{Sample Size and Power Calculation}

Sample size is calculated based on the primary outcomes: 1) scores of behavioral assessment questionnaires, including the ABC and SRS-2; 2) scores of objective ASD behavioral tests, including eye 
tracking and emotional face matching tests; 3 ) serum OXT changes. To achieve statistical power of $80 \%$ with overall one-sided significance level of alpha $=0.10$, if we assume a large effect size of 0.8 (Cohen's d) in each of primary outcomes for the effect of probiotics alone vs probiotics plus OXT therapy at the first phase (12 weeks) of this pilot study, an estimated number of 26 ASD patients in each group would be needed based on independent two-sample t test with Bonferroni correction for multiple comparison. If a potential non-compliance or dropout rate of $10 \%$ is assumed, we plan to enroll 60 autistic subjects in total for this pilot study. Sample size was carried out using PASS 16 Power Analysis and Sample Size Software (NCSS, LLC. Kaysville, Utah).

\section{Statistical Analysis}

First, descriptive statistics will be provided to characterize recruited patients at baseline for treatment and control groups and ensure that balance is achieved by randomization. The presentation of the data will follow the CONSORT recommendations for reporting results of RCTs. Statistical procedures carried out use $a=0.10$ as significance level. All analyses will be performed using SAS, version 9.4 (SAS Institute, Inc.).

Analyses based on the intent-to-treat principle are designed to examine the treatment effect of probiotics vs placebo at the end of first stage of trial on primary outcomes, which are behavioral scores (i.e., ABC and SRS-2), behavioral testing results (i.e., eye tracking and emotional face matching tests), and serum OXT changes. Since there are multiple primary outcomes, false discovery rate due to multiple comparisons will be taken into account. For primary aim 1, independent two-sample unpaired t test will be conducted to study the effect of L. reuteri versus placebo on primary outcomes at 12 weeks. For primary aim 2, the difference of primary outcomes between 12 and 24 weeks will be calculated first, and independent two-sample unpaired $t$ test will be performed on the calculated difference to examine whether OXT therapy enhances the treatment effect of L. reuteri.

Per-protocol analyses for secondary outcomes will be performed based on available data due to relatively small sample size, especially the subsample in fMRI study. Methods for secondary analyses conducted will be similar to primary analyses. Two-sample t tests will be performed on secondary outcomes as appropriate.

Gut microbiome data will be analyzed with QIIME software package v. 2018.2.027. For short chain fatty acid profiling, data integrity will be checked and results will be examined for outliers and detectability. Volcano plots will be generated displaying fold differences vs. significance adjusted for multiple testing. As many significant short chain fatty acids will be correlated with each other, we will employ data reduction techniques such as hierarchical cluster analysis from which leading fatty acid metabolites in each cluster will be tested for independence and non-redundancy by means of regression models. Twosample t tests will be performed on outcomes, such as microbial relative abundances, alpha-diversity, and leading short chain fatty acid metabolites as appropriate.

\section{Follow Up and Compliance Assessment}


Follow-up evaluations will include compliance evaluation and safety assessments of potential adverse effects. Compliance will be assessed on a monthly basis via telephone check-in. Compliance to intervention will be also assessed by determining the number of capsules returned at each follow-up visit at week 12 and week 24 from the treatment packs dispensed as well as post-intervention changes of serum OXT levels. Adverse reactions will be monitored on a monthly basis via self-report and telephone check-in, if guardians fail to complete online surveys. All adverse events will be reported to the Human Research Committee promptly in accordance with guidelines. Randomized patients will have to be immediately withdrawn from the trial if they complain serious adverse events.

\section{Data Monitoring}

The Principal Investigator and Co-Investigators will monitor data as it is acquired to ensure the quality of the data. Data monitoring and auditing will be performed on a monthly basis on RedCap.

\section{Ethical Considerations}

The current protocol has received IRB approval at Massachusetts General Hospital, and any potential amendments will be submitted for evaluation in a timely manner. All studies will be performed with the approval of Partners Human Research Committee. Informed consent for the study will be obtained inperson at the initial study visit, after inclusion criteria have been met and prior to any experimental procedure. This will be conducted in a private room by a Physician Investigator. The investigator will explain the purpose, procedures, and potential discomforts and risks of the study before obtaining signed informed consent and assent from one parent and, if applicable, from the prospective subject. Subjects 7-13 years old will sign a separate assent form and subjects 14-15 years old will give assent on the consent form. Subjects will receive copies of the consent and assent forms.

There is potential for loss of confidentiality by participating in this study. Every effort will be made to protect the confidentiality of identifiable information. Subjects may benefit from probiotics and oxytocin treatment with respect to a positive change of their behavioral and Gl symptoms. Patients and their primary care physicians/pediatricians will receive reports of their gut microbiome, and neuroinjury/neurotransmitter/inflammatory markers, if any actionable abnormalities are identified during the study.

\section{Access to Data}

All information regarding experimental patients will be kept in a file cabinet in the office of the Principal Investigator. All data for presentation will be identified by a code number only. All data spreadsheets will be housed on the password protected server at the lab and de-identified as described above. All MGH collaborators will have access to the de-identified data as needed for analysis and manuscript preparation.

\section{Post-trial Care}


We do not plan to provide post-trial care, but all participants will receive 3 months of probiotics free supply (regardless of original group) at the conclusion of the study period. We will continue to follow up with adverse reactions during this post-trial period.

\section{Interim Analysis and Stopping Guideline}

We plan to conduct interim analysis at 12 weeks. We will stop the study if: treatment with probiotics is found to be convincingly not different compared to placebo; with no improvement in primary, secondary outcomes; adverse effects or toxicities are too severe; data quality is poor; accrual is slow; adherence to treatment is unacceptably low; or if study integrity has been undermined by fraud or misconduct.

\section{Dissemination Policy}

We will disseminate details of the trial design and its results through publication in peer-reviewed journals and reporting on Clinicaltrial.gov.

\section{Discussion}

This study is the first parallel RCT to investigate the effect of a psychotropic probiotics strain on ASD social-behavioral symptoms, with an emphasis on OXT signaling pathway. Although multiple OXT-related ASD clinical trials have been conducted, this is also the first clinical study designed to investigate synergistic relationship between probiotics and exogenous OXT administration in an autism treatment. Further, to better our mechanistic understanding of possible effects of L. reuteri and OXT on ASD social functions, we plan to further explore the effects of L. reuteri and OXT on biomarkers and indices of autonomic nervous system, inflammatory processes, and neurotransmitters/circuits.

We include both subjective and objective social-behavioral tests to quantify different aspects of ASD behavior as primary outcomes. Of note, a new video-based eye tracking device developed by Kong et al. ${ }^{26}$ could assess fixation/attention to human body parts such as the mouth and regions around the eye. The test can achieve sensitivity of $93 \%$ and specificity $86 \%$ in distinguishing ASD and non-ASD individuals ${ }^{24}$. In addition, an emotional facial matching paradigm has be used to objectively assess ASD patients' ability to detect and characterize human emotions in another ASD drug trial ${ }^{25}$.

Based on our currently mechanistic understanding, the roles of L. reuteri and OXT in OXT signaling/gutbrain axis may involve the autonomic nervous system, the immune system, and other OXT-related neurotransmitter ${ }^{14,15}$. In our secondary aim, we proposed to explore whether serum neuroinflammatory/neurotransmitter/imaging biomarkers, autonomic functions, and gut microbiome may be altered by L. reuteri and OXT treatment, and whether their baseline levels may predict the treatment response to either L. reuteri therapy alone or L. reuteri plus OXT therapy.

Apart from OXT levels, a panel of several other biomarkers is proposed as secondary outcomes. Probiotics affects the gut brain axis through the immune system; a recent systematic review suggest 
cytokine aberrations in ASD patients, such as IL-1 beta, IL-6, TGF-beta and Interferon-gamma ${ }^{28}$. They might correlate with markers that directly signify neuronal/glial injury such as $\mathrm{S} 100 \mathrm{~B}^{29,30}$, myelin basic protein (MBP) $)^{31}$ and glial fibrillary acidic protein (GFAP) ${ }^{32}$.

Several other neurotransmitters have been implicated in the pathophysiology of ASD, including GABA, serotonin, and dopamine ${ }^{19,20,33}$. All of them are also known to interact with OXT signaling pathway. Levels of platelet-free plasma GABA are significantly elevated in ASD patients. Specifically, high blood plasma levels of GABA are associated with reduced GABA levels in the ASD brain, which leads to the hypothesis that imbalance in GABA would contribute to social deficits commonly seen in ASD patients. Further, animal studies showed that introduction of oxytocin to the ASD mice is capable of alleviating social deficits by restoring an interrupted developmental switch from GABA as an excitatory neurotransmitter to an inhibitory neurotransmitter ${ }^{33}$. Derangement in the serotonin system is proposed to play a role in ASD due to its pleiotropic effects across multiple neuronal systems throughout development, and elevated whole blood serotonin is present in more than $25 \%$ of ASD children ${ }^{21}$. OXT may inhibit serotonin signaling ${ }^{19}$, consistent with the finding that OXT and whole blood 5-HT levels in ASD subject consistently display a negative correlation ${ }^{23}$. Conversely, OXT and Dopamine may have synergistic functions, as OXT facilitates dopamine release in particularly the midbrain striatal nucleus accumbens, mediating reward processing related to social-functions ${ }^{20}$. Similar to OXT, plasma Dopamine is decreased in ASD children ${ }^{34}$, although it is not clear whether baseline Dopamine level/signaling is decreased at the neuronal level in patients with ASD. Other neurotransmitters that are altered in ASD including norepinephrine and epinephrine (both elevated compared to controls). They are important mediators of the autonomic nervous system, and assessing their serum levels will complement the direct measurement of autonomic indices using the wristband.

We hope that this pilot study will serve as a starting point to address any potential roles of probiotics induced endogenous OXT in ASD. If significant correlations are found, we would like to confirm the results of the pilot study on a larger scale. The results from this pilot study will provide important proofof-concept information that will serve as the basis for further scientific studies. We hope that information generated by our research will help to assess and promote the development of effective therapeutic interventions to treat the behavioral symptoms in ASD patients.

\section{Abbreviations}

$A B C$ : aberrant behavior checklist; ADI-R: autism diagnostic interview-revised; ADOS: autism diagnostic observation schedule; ASD: autism spectrum disorders; BOLD: blood oxygen level dependent; BVP: blood volume pulse; CGI-I: clinical global impression-improvement; CONSORT: Consolidated Standards of Reporting Trials; DSM: Diagnostic and Statistical Manual of Mental Disorders; EDA: electrodermal activity; GABA: oxytocin-mediated neuroprotective $\gamma$-aminobutyric acid; Gl: gastrointestinal; HRV: heart rate variability; IL-1: interleukin 1; IL-6: interleukin 6; MBP: myelin basic protein; GFAP: glial fibrillary acidic 
protein; OXT: oxytocin; RCT: randomized controlled trials; SAR: specific absorption rate; SRS: social responsiveness scale.

\section{Declarations}

The study was approved by the Partners Human Research Committee.

\section{Ethics approval and consent to participate}

The trial will be overseen by an independent trial steering committee and a separate data and safety monitoring committee. All the participants will provide written informed consent. The study design, including the screening, randomization, and follow-up of ASD patients, was approved by Massachusetts General Hospital (IRB protocol number: 2017P001667).

\section{Consent for publication}

The manuscript does not contain any identifiable individual person's data in any form.

\section{Availability of data and material}

All manuscripts must include an 'Availability of data and materials' statement. Data availability statements should include information on where data supporting the results reported in the article can be found including, where applicable, hyperlinks to publicly archived datasets analyzed or generated during the study. By data we mean the minimal dataset that would be necessary to interpret, replicate and build upon the findings reported in the article. We recognize it is not always possible to share research data publicly, for instance when individual privacy could be compromised, and in such instances data availability should still be stated in the manuscript along with any conditions for access.

\section{Trial status}

IRB protocol number 2017P001667, 26 July 2018. Recruitment began on 12 July 2018. At the time of submission of this paper, patient recruitment is still ongoing. Recruitment will complete approximately on 1 September 2019.

\section{Authors' contributions}

XJK conceived and designed the study with input from KK; XJK led IRB and clinical trial application with assistance from MK, JG, ZJS; XJK modified the protocol with input from JG, ZJS, JuL; XJK, JuL, and JiL wrote the first draft of the manuscript, with significant guidance from YQS and input from PS; JuL, JiL and YQS contributed to data analysis plan. All authors have read and approved on this version of manuscript.

\section{Competing interests}


The authors declare that they have no competing interests.

\section{Acknowledgements}

We are grateful for Drs. Susan Erdman, Elizabeth Austin Lawson, Nouchine Hadjikhani, Cunjian Dong, Pheobe Chen, Bruce Rosen for their generous contribution and suggestions.

\section{Funding}

This study is funded by internal grants 233263 (XJK) and 230361 (XJK) from Massachusetts General Hospital. The funding sponsor has no role in the study design, data collection, management, data analysis and interpretation, or writing of the report.

\section{Author details}

Xue-Jun Kong, MD, xkong1@mgh.harvard.edu, Athinoula A. Martinos Center for Biomedical Imaging, Massachusetts General Hospital, $14913^{\text {th }}$ Street, Charlestown, MA;

Jun Liu, PhD, jun_liu@hms.harvard.edu, Athinoula A. Martinos Center for Biomedical Imaging, Massachusetts General Hospital, Harvard Medical School, Boston, MA;

Jing Li, MS, jl204@iu.edu, Department of Biostatistics, Richard M. Fairbanks School of Public Health, Indiana University, Indianapolis, IN;

Kenneth Kwong, PhD, kkwong@partners.org, Athinoula A. Martinos Center for Biomedical Imaging, Massachusetts General Hospital ;

Madelyn Koh, BS, mkoh@mgh.harvard.edu, Athinoula A. Martinos Center for Biomedical Imaging, Massachusetts General Hospital;

Jason J. Guo, PhD, j.guo@northeastern.edu, Barnett Institute for Chemical and Biological Analysis, Department of Chemistry and Chemical Biology, Northeastern University, Boston, MA;

Piyawat Sukijthamapan, BS, piyawat_sukijthamapan@hms.harvard.edu, Harvard Medical School, Boston, MA;

Zhenyu Jim Sun, PhD, jim_sun@dfci.harvard.edu, Dana Farber Cancer Institute, Harvard Medical School, Boston, MA;

Yiqing Song, MD, ScD, yiqsong@iu.edu, Department of Epidemiology, Richard M. Fairbanks School of Public Health, Indiana University, Indianapolis, IN.

\section{References}


1. Baio J, Wiggins L, Christensen DL, Maenner MJ, Daniels J, Warren Z, et al. Prevalence of autism spectrum disorder among children aged 8 years-Autism and Developmental Disabilities Monitoring Network, 11 Sites, United States, 2014. MMWR Surveillance Summaries. 2018;67(6):1.

2. Mayer EA, Padua D, Tillisch K. Altered brain-gut axis in autism: Comorbidity or causative mechanisms? Bioessays. 2014;36(10):933-9.

3. Alam R, Abdolmaleky HM, Zhou Jr. Microbiome, inflammation, epigenetic alterations, and mental diseases. American Journal of Medical Genetics Part B: Neuropsychiatric Genetics. 2017;174(6):65160.

4. Stigler KA, Sweeten TL, Posey DJ, McDougle CJ. Autism and immune factors: a comprehensive review. Research in Autism Spectrum Disorders. 2009;3(4):840-60.

5. Erdman S, Poutahidis T. Microbes and oxytocin: benefits for host physiology and behavior. International review of neurobiology. 131: Elsevier; 2016. p. 91-126.

6. Pobbe RL, Pearson BL, Defensor EB, Bolivar VJ, Young III WS, Lee H-J, et al. Oxytocin receptor knockout mice display deficits in the expression of autism-related behaviors. Hormones and behavior. 2012;61(3):436-44.

7. Teng BL, Nikolova VD, Riddick NV, Agster KL, Crowley JJ, Baker LK, et al. Reversal of social deficits by subchronic oxytocin in two autism mouse models. Neuropharmacology. 2016;105:61-71.

8. Husarova VM, Lakatosova S, Pivovarciova A, Babinska K, Bakos J, Durdiakova J, et al. Plasma oxytocin in children with autism and its correlations with behavioral parameters in children and parents. Psychiatry investigation. 2016;13(2):174-83.

9. Neumann ID. Brain oxytocin: a key regulator of emotional and social behaviours in both females and males. Journal of neuroendocrinology. 2008;20(6):858-65.

10. Stavropoulos KK, Carver LJ. Research review: social motivation and oxytocin in autism-implications for joint attention development and intervention. Journal of Child Psychology and Psychiatry. 2013;54(6):603-18.

11. Ooi YP, Weng S-J, Kossowsky J, Gerger H, Sung M. Oxytocin and autism spectrum disorders: a systematic review and meta-analysis of randomized controlled trials. Pharmacopsychiatry. 2017; 50(01):5-13.

12. Andari E, Duhamel J-R, Zalla T, Herbrecht E, Leboyer M, Sirigu A. Promoting social behavior with oxytocin in high-functioning autism spectrum disorders. Proceedings of the National Academy of Sciences. 2010;107(9):4389-94.

13. Poutahidis T, Kearney SM, Levkovich T, Qi P, Varian BJ, Lakritz JR, et al. Microbial symbionts accelerate wound healing via the neuropeptide hormone oxytocin. PloS one. 2013;8(10):e78898.

14. Buffington SA, Di Prisco GV, Auchtung TA, Ajami NJ, Petrosino JF, Costa-Mattioli M. Microbial reconstitution reverses maternal diet-induced social and synaptic deficits in offspring. Cell. 2016;165(7):1762-75.

15. Sgritta M, Dooling SW, Buffington SA, Momin EN, Francis MB, Britton RA, et al. Mechanisms Underlying Microbial-Mediated Changes in Social Behavior in Mouse Models of Autism Spectrum 
Disorder. Neuron. 2018.

16. Varian BJ, Poutahidis T, DiBenedictis BT, Levkovich T, Ibrahim Y, Didyk E, et al. Microbial lysate upregulates host oxytocin. Brain, behavior, and immunity. 2017;61:36-49.

17. Tillisch K, Labus J, Kilpatrick L, Jiang Z, Stains J, Ebrat B, et al. Consumption of fermented milk product with probiotic modulates brain activity. Gastroenterology. 2013;144(7):1394-401. e4.

18. Pinto-Sanchez MI, Hall GB, Ghajar K, Nardelli A, Bolino C, Lau JT, et al. Probiotic Bifidobacterium longum NCC3001 reduces depression scores and alters brain activity: a pilot study in patients with irritable bowel syndrome. Gastroenterology. 2017;153(2):448-59. e8.

19. Sung V, D’Amico F, Cabana MD, Chau K, Koren G, Savino F, et al. Lactobacillus reuteri to treat infant colic: a meta-analysis. Pediatrics. 2018;141(1):e20171811.

20. Fatheree NY, Liu Y, Taylor CM, Hoang TK, Cai C, Rahbar MH, et al. Lactobacillus reuteri for infants with colic: a double-blind, placebo-controlled, randomized clinical trial. The Journal of pediatrics. 2017;191:170-8. e2.

21. Gutierrez-Castrellon P, Lopez-Velazquez G, Diaz-Garcia L, Jimenez-Gutierrez C, Mancilla-Ramirez J, Estevez-Jimenez $\mathrm{J}$, et al. Diarrhea in preschool children and Lactobacillus reuteri: a randomized controlled trial. Pediatrics. 2014:peds. 2013-0652.

22. Parker KJ, Oztan O, Libove RA, Sumiyoshi RD, Jackson LP, Karhson DS, et al. Intranasal oxytocin treatment for social deficits and biomarkers of response in children with autism. Proceedings of the National Academy of Sciences. 2017;114(30):8119-24.

23. Yatawara C, Einfeld S, Hickie I, Davenport T, Guastella A. The effect of oxytocin nasal spray on social interaction deficits observed in young children with autism: a randomized clinical crossover trial. Molecular psychiatry. 2016;21(9):1225.

24. Wan G, Kong X, Sun B, Yu S, Tu Y, Park J, et al. Applying Eye Tracking to Identify Autism Spectrum Disorder in Children. Journal of autism and developmental disorders. 2018:1-7.

25. Hadjikhani N, Zürcher NR, Rogier O, Ruest T, Hippolyte L, Ben-Ari Y, et al. Improving emotional face perception in autism with diuretic bumetanide: a proof-of-concept behavioral and functional brain imaging pilot study. Autism. 2015;19(2):149-57.

26. Kong X, Wang BK, Park J, Kong J. Introduction of a New Video-Based Eye Tracking Paradigm for Early Detection of ASD. North American Journal of Medicine and Science. 2017;10(4).

27. Caporaso JG, Kuczynski J, Stombaugh J, Bittinger K, Bushman FD, Costello EK, et al. QIIME allows analysis of high-throughput community sequencing data. Nature methods. 2010;7(5):335.

28. Masi A, Quintana D, Glozier N, Lloyd A, Hickie I, Guastella A. Cytokine aberrations in autism spectrum disorder: a systematic review and meta-analysis. Molecular psychiatry. 2015;20(4):440.

29. Guloksuz SA, Abali O, Aktas Cetin E, Bilgic Gazioglu S, Deniz G, Yildirim A, et al. Elevated plasma concentrations of $\mathrm{S} 100$ calcium-binding protein B and tumor necrosis factor alpha in children with autism spectrum disorders. Revista Brasileira de Psiquiatria. 2017;39(3):195-200. 
30. Al-Ayadhi LY, Mostafa GA. A lack of association between elevated serum levels of S100B protein and autoimmunity in autistic children. Journal of neuroinflammation. 2012;9(1):54.

31. Esnafoglu E, Ayyıldız SN, Cırrık S, Erturk EY, Erdil A, Daglı A, et al. Evaluation of serum Neuron-specific enolase, $\mathrm{S} 100 \mathrm{~B}$, myelin basic protein and glial fibrilliary acidic protein as brain specific proteins in children with autism spectrum disorder. International Journal of Developmental Neuroscience. 2017;61:86-91.

32. Laurence J, Fatemi S. Glial fibrillary acidic protein is elevated in superior frontal, parietal and cerebellar cortices of autistic subjects. The Cerebellum. 2005;4(3):206-10.

33. Launay J-M, Bursztejn C, Ferrari P, Dreux C, Braconnier A, Zarifian E, et al. Catecholamines metabolism in infantile autism: a controlled study of 22 autistic children. Journal of Autism and Developmental Disorders. 1987;17(3):333-47.

34. Nicolucci AC, Hume MP, Martínez I, Mayengbam S, Walter J, Reimer RA. Prebiotics reduce body fat and alter intestinal microbiota in children who are overweight or with obesity. Gastroenterology. 2017;153(3):711-22.

\section{Figures}




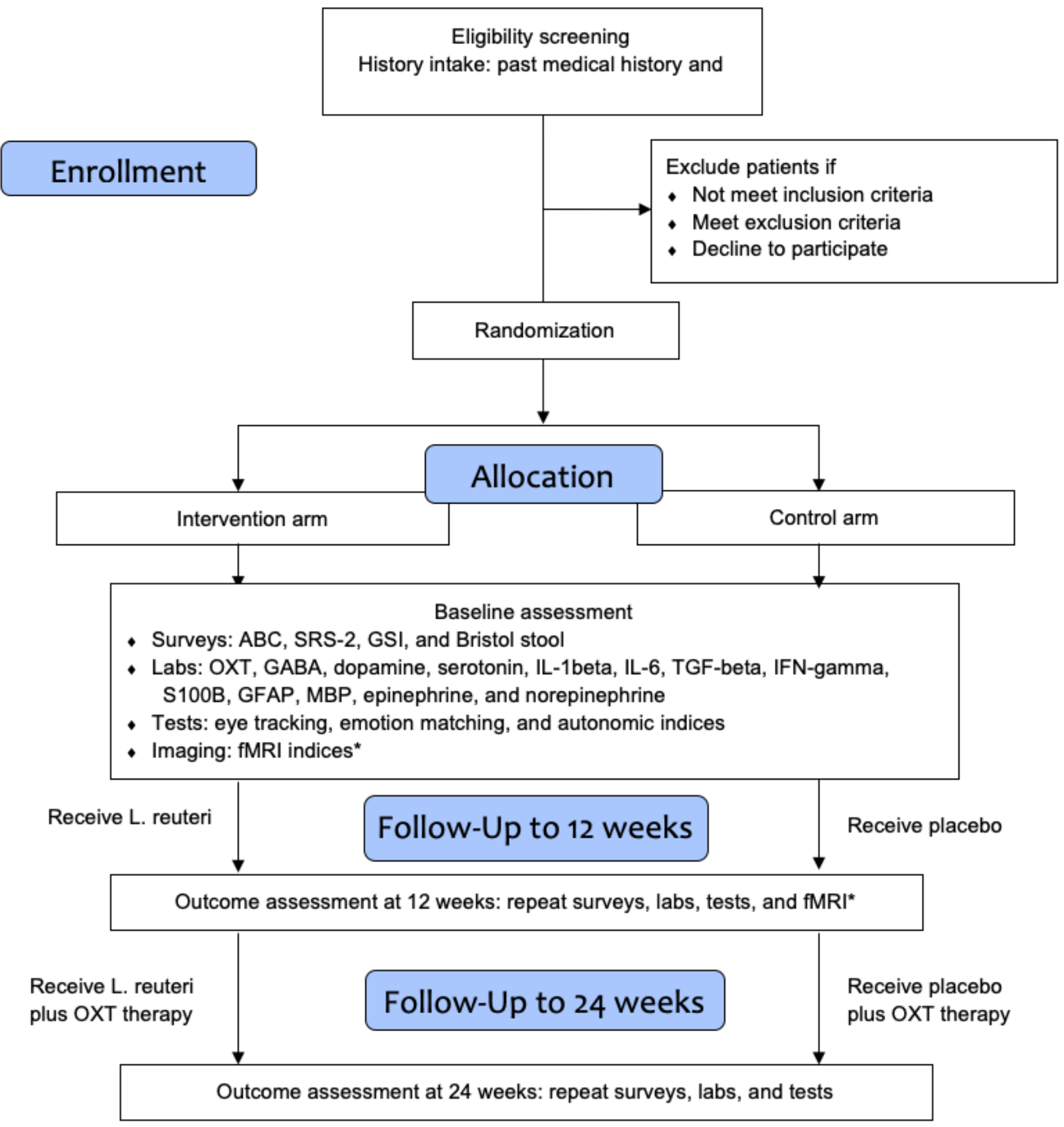

*For fMRI-eligible participants only

\section{Figure 1}

Study design. Abbreviations: OXT: oxytocin, ABC: Aberrant Behavior Checklist, SRS: Social Responsiveness Scale, GSI: Gastrointestinal Severity Index, GABA: gamma-aminobutyric acid, IL: interleukin, TGF: tumor growth factor, IFN: interferon, GFAP: glial fibrillary acidic protein, MBP: myelin basic protein, TNF: tumor necrosis factor, fMRI: functional magnetic resonance imaging, L. reuteri: 


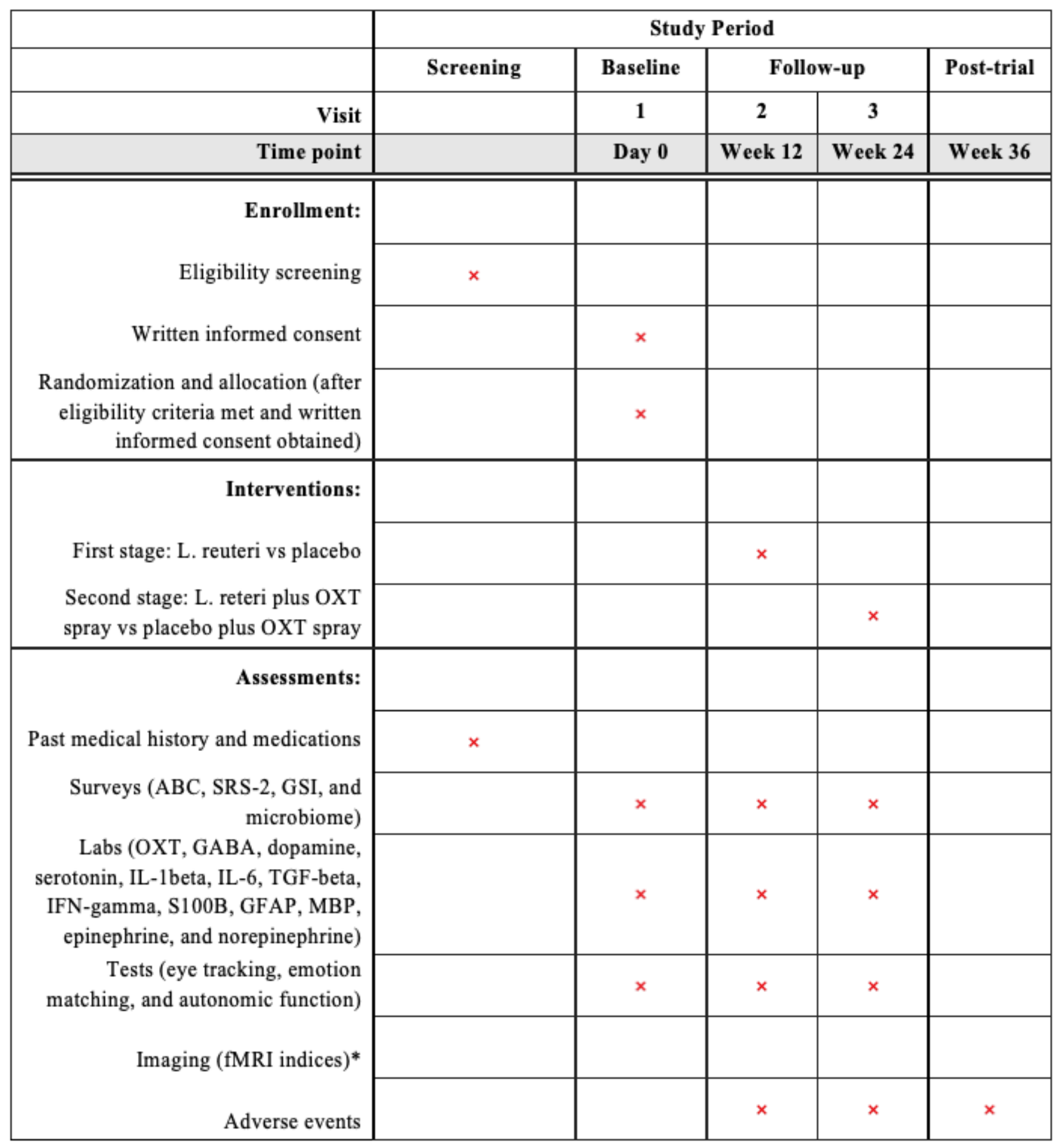

*For fMRI-eligible participants only

\section{Figure 2}

Study schedule of enrollment, interventions, and assessments. Abbreviations: OXT: oxytocin, ABC: Aberrant Behavior Checklist, SRS: Social Responsiveness Scale, GSI: Gastrointestinal Severity Index, GABA: gamma-aminobutyric acid, IL: interleukin, TGF: tumor growth factor, IFN: interferon, GFAP: glial fibrillary acidic protein, MBP: myelin basic protein, TNF: tumor necrosis factor, fMRI: functional magnetic resonance imaging, L. reuteri: Lactobacillus reuteri 


\section{Supplementary Files}

This is a list of supplementary files associated with this preprint. Click to download.

- supplement1.doc 